\title{
Personality Profile of Patients With Chronic Fatigue SYNDROME
}

Olivier Le Bon, Bernard Cappeliez, Daniel Neu, Luc Stulens, Guy Hoffmann, Michel Hansenne, Luc Lambrecht, Marc Ansseau \& Paul Linkowski

Olivier Le Bon, Bernard Cappeliez, Daniel Neu, Luc Stulens, Guy Hoffmann, and Paul Linkowski are affiliated with Brugmann University Hospital, Department of Psychiatry, Université Libre de Bruxelles, Belgium.

Michel Hansenne and Marc Ansseau are affiliated with University Hospital Liège, Department of Psychiatry, Université de Liège, Belgium.

Luc Lambrecht is affiliated with University Hospital Gent, Department of Internal Medicine, Gent, Belgium. Address correspondence to: Olivier Le Bon, CHU Brugmann Service de Psychiatrie, Plaza A. Van Gehuchten, 1020 Brussels, Belgium (E-mail: olivier.lebon@chu-brugmann.be).

KEYWORDS: Personality, chronic fatigue syndrome, TCI

\section{ABSTRACT}

Personality may play a role in the predisposition, the precipitation and/or the maintenance of the CFS. Thirty-six consecutively examined female patients hospitalised for a sleep workup, filled out a Temperament and Character Inventory (TCI) questionnaire. A MANOVA compared the patients with a control group of females matched for age. Significant scores were obtained for dimensions such as Harm Avoidance, Reward Dependence, and Self-Directedness. However, the only subdimension of Harm Avoidance that proved significantly higher in CFS than in controls was "Fatigability," which is likely to overlap with the core CFS symptom. All in all, the personality structure does not appear to play a major role in the CFS. 


\section{Introduction}

Chronic fatigue syndrome (CFS) is a severe illness, affecting between 2 and 130 patients per 100,000 according to an estimate carried out in a primary care centre (Wessely et al., 1996; also see Gallagher et al., 2004; Furberg et al., 2005; Weir et al., 2006). By its present definition (Center for Disease Control (CDC) criteria; Fukuda et al., 1994), CFS includes abnormal fatigue for more than 6 months, numerous somatic complaints, and neuropsychological disorders, as well as a reduction in work, social, and personal activity. The etiology of CFS is unknown and hypotheses run from viral infection to immunity to sleep and to psychiatric disorders, including stress (for a review on etiology, see Fischler, 1999; Maquet et al., 2006; Cho et al., 2006). As CFS is comorbid more often than not with several disorders-from Fibromyalgia to Raynaud, to Generalized Anxiety Disorder and sleep disorders to cite a few-its mere existence is still harshly debated. There remains considerable controversy over whether the comorbid psychiatric diagnoses are a primary feature of the syndrome or a secondary manifestation of its debilitating main symptom. Unicity of the syndrome is another issue: as is often the case with medical entities defined by their core symptom, the question arises of whether the syndrome represents distinct phenomena ("split") or one basic disorder with several forms and connections("lump")(Van Hoof and De Meirleir, 2005a). For all these issues, the CFS is presently no more than a descriptive, consensus diagnosis.

Most of these questions remain unresolved nowadays but it definitely remains interesting to further explore the psychiatric side of CFS. Psychopathological hypotheses on CFS are generally focused on its frequent association with psychiatric clinical disorders. We have shown previously a prevalence of 50\% Generalized Anxiety, 13\% Major Depression, and 13\% Panic Disorder in a patients sample suffering from CFS (Le Bon et al., 2000). The study of personality is another option, as it could predispose, precipitate, or maintain CFS. Personality can be studied by category, as proposed in the DSM (axis-II) (APA, 1994), or dimensionally with various questionnaires. The category diagnostics possess the advantage of isolating psychopathological groups, which can be useful for research. They, however, probably differentiate too distinctly between the normal and the pathological, while the dimensional profiles show a continuity between these two positions (Frances, 1982). In dimensional studies, a small minority of control subjects show average scores simultaneously on all personality dimensions (Le Bon et al., 1998), so that the common situation is to present profiles with at least one, if not more, dimensions sometimes largely deviating from the mean. The more deviating dimensions a profile includes, the more it will define original, but perhaps more problematic, personalities. Some personality patterns have been linked to DSM axis-II categories (Cloninger, 1987). Particular combinations maybe more likely to be associated with specific mental disorders.

Relatively few studies to date tried to define the personality profiles of CFS patients (Millon et al., 1989; Pepper et al., 1993; Russo et al., 1994; Schmaling and Jones, 1996; Johnson et al., 1996; Van Hoof and De Meirleir, 2005b) and in general, dramatic or emotional traits are noted in these patients (Johnson et al., 1996). However, many studies suffer from methodological weaknesses, such as a lack of control group or criteria to define CFS other than those of the CDC. A controlled study showed CFS patients to have more DSM axis-II Personality Disorders and more 
neuroticism than controls (Johnson et al., 1999). In that study, a comparison was performed with patients with Multiple Sclerosis, a disorder with proven neurological origin and patients from both conditions showed approximately the same profiles. White et al. (2000) showed a higher level of Perfectionism, Doubts before Action and Concern over Mistakes (Multidimensional Perfectionism Scale; Frost et al., 1990) as well as Poor Self-Esteem (Rosenberg Self-Esteem Scale; Rosenberg, 1965).

Human personality is the result of a combination of genetic, environmental, and developmental factors. Taking this complexity into account, the models built by Cloninger et al. $(1986,1987$, 1993) integrate these dimensions, whereas most other personality models and scoring scales almost exclusively rely on biology (Eysenck, 1967, 1990) or do not refer to biology at all (most of the others). The Cloninger biosocial model is thus particularly attractive from a medical point of view. It includes four Temperament dimensions, postulated to be inheritable and independent, and which were grouped in the original questionnaire (Tridimensional Personality Questionnaire, TPQ, Cloninger, 1987). Three Character dimensions were later added (Cloninger et al., 1993). These are supposedly learned and not linked to particular biological processes. The present model thus includes seven dimensions.

The Temperament dimensions include (1) Novelty Seeking (NS), supposedly associated with dopaminergic activity, was defined as the tendency to respond actively to novel stimuli leading to the pursuit of rewards and escape from punishment; (2) Harm Avoidance (HA), linked to serotonergic activity, corresponds to the tendency toward an inhibitory response to signals of aversive stimuli leading to avoidance of punishment and nonreward; (3) Reward Dependence (RD), associated with noradrenergic activity, was defined as the tendency for a positive response to signals of reward to maintain or resist behavioral extinction; (4) Persistence (PE), originally included in the RD dimension, was later individualized and is not presently specifically linked to a neurotransmitter. The Character dimensions include (5) Self-Directedness (SD) refers to the ability of an individual to control, regulate and adapt his or her behavior to fit the situation in agreement with individually chosen goals and values; Cooperativeness (CO) was formulated to account for individual differences in identification with and acceptance of other people. Cooperative individuals are described as socially tolerant, empathic, helpful, and compassionate, whereas uncooperative individuals are described as socially intolerant, uninterested in other people, unhelpful, and revengeful; Self-Transcendence (ST) is a character associated with spirituality and refers generally to identification with everything conceived as essential and consequential parts of a unified whole.

A study using TPQ (Christodoulou et al., 1999) showed higher levels of Harm Avoidance and a lesser degree of Reward Dependence in CFS than in control subjects and demonstrated that their personality profile was similar to that of Multiple Sclerosis patients. The present study replicates the comparison between CFS and controls for the Temperament dimensions and extends it to dimensions of Character, by using the Temperament and Character Inventory (TCI), a 226-items, binary, forced choice, well-validated self-questionnaire, developed to assess the seven dimensions of personality (Cloninger et al., 1994). 


\section{PATIENTS AND CONTROLS}

The study sample consisted of 36 female patients aged 20-58, consecutively admitted for a medical and sleep workup for chronic fatigue, in a University hospital (CHU Brugmann, Belgiumtertiary care). Inclusion criteria were those of CDC (Fukuda et al., 1994): (1) clinically evaluated chronic fatigue, of unknown or undetermined origin, with a recent or precise past beginning, not being the result of physical exhaustion, not improved by rest and which causes a substantial reduction when compared with past levels of social, occupational, or personal activity; (2) simultaneous presence of four or more of the following symptoms, each of which having persisted for six consecutive months or more and were not present before the onset of fatigue(reduction reported by the person of short-term memory or concentration; throat irritation; sensitivity of cervical or axillary lymph glands; muscular pain; multiple articular pains without inflammation or erythema; headaches of a type, localisation or severity differing from previous headaches; nonrepairing sleep; post-physical exercise discomfort lasting longer than 24 hours); (3) exclusion of any present or past persistent medical pathology which could explain chronic fatigue; exclusion of some psychiatric pathologies (major depressive problems with psychotic or melancholic characteristics; bipolar thymic disorder; schizophrenia (all subtypes); all delirium disorders; dementia; eating disorders; substance abuse in the two years preceding installation of the syndrome; severe obesity.

In addition, primary sleep disorders (respiratory sleep disorder, periodic limb movements, narcolepsy, idiopathic excessive sleepiness) have been excluded based on the criteria of the American Sleep Disorders Association (1997). All psychotropes must have been interrupted minimum fifteen days before the passage of the questionnaires.

\section{PROCEDURE}

TCI questionnaires, and Hamilton scales for Depression (17 items) and Anxiety, were given to the patients at the end of their two-day stay in the sleep unit of Brugmann Hospital. Control subjects were informed by mail that a personality questionnaire would be added to the usual material of the inquiry. The questionnaires were mailed 15 days after the instructions. An interviewer went to the subjects' residences to collect the questionnaires and to check whether they were adequately completed. Subjects and patients were required to indicate the answer that applied the most to them, even if answering was difficult. No missing items were accepted.

Seventy-two female control subjects originate from a normative TCI databank $(\mathrm{n}=322)$ representative of the Belgian population with respect to sex, age, geographical area, and educational level (Hansenne et al., 2001). The sample was selected in order to obtain mean values for age (a maximum of two years difference between subjects and patients) and educational level comparable with those of the patients. The subjects completed the questionnaire as part of the 1997 family survey of the University of Liège. This survey has been conducted on a nearly annual basis since 1992, to evaluate a series of variables on family life. The subjects were informed by mail that a personality questionnaire would be added to the usual material of the inquiry. The questionnaires were mailed 15 days after the instructions. An interviewer went to the subjects' residences to collect the questionnaires and to check whether these were adequately completed. 


\section{STATISTICS}

The distribution was tested using Kolmogorov-Smirnov tests and was found normal for all the dimensions (NS: $\mathrm{z}=.596, \mathrm{p}=.870 ; \mathrm{HA}: \mathrm{z}=.623, \mathrm{p}=.833$; RD: $\mathrm{z}=.800, \mathrm{p}=.544 ; \mathrm{PE}: \mathrm{z}=.943, \mathrm{p}$ $=.336 ;$ ST: $z=.690, p=.728 ;$ CO: $z=.695, p=.720 ;$ ST: $z=.598, p=.867$ ). The group comparisons were achieved by MANOVA. Comparisons for each variable was achieved by Student t-tests for unpaired groups. Comparisons between categories was performed with ChiSquare. The hypotheses tests were bilateral and achieved to degree of significance of $5 \%$. The statistics were performed using SPSS 13 (SPSS Inc.).

\section{RESULTS}

\section{DESCRIPTIVE DATA}

The average age of the patients group was 37.2 years ( \pm 8.6 ), while the patient group was 38.1. $( \pm 7.7)$. The age comparison between groups was not significant. Table 1 shows descriptive data on cases with Sudden Onset, Work Reduction, Married or Stable Couple, comorbidity with Fibromyalgia, Major Depressive Disorder, Generalized Anxiety Disorder, Phobias (all types), Panic Disorder, Post-Traumatic Stress Disorder, Any Psychiatric Disorder, Raynaud, and Irritable Bowel. Education level was: university: 5 (15\%); higher nonuniversity education: 6 (17\%); technical: 15 (44\%); worker: 3 (9\%); no particular skill: 5 (15\%) (2 missing values). No difference in education level was observed between the groups.

\section{DATA ANALYSES}

Within the CFS group, the dimensions were compared for each of the factors described above, from a descriptive perspective, except for Panic Disorder, PTSD, and Irritable Bowel, where the sample was too reduced for statistical comparisons. The only significant difference $(p=.024)$ was when Self-Self-Directedness was split by "Any Psychiatric Disorder." The scores were 30.6 (SD: 7.6) (present) versus 37.0 (SD: 7.0) (absent). Descriptively again, the associations between dimensions, and depression and anxiety severity were measured and no significant correlation was found. As no other difference was noted, comparisons with control subjects were performed for CFS patients as a whole, CFS patients with psychiatric disorders, and CFS patients without psychiatric disorders (Table 2). 
TABLE 1. Descriptive data.

\begin{tabular}{lcccc}
\hline & (n) & (n) & Missing (n) & Percentage \\
\hline Sudden Onset & 7 & 27 & 2 & 19 \\
Work Reduction & 17 & 17 & 2 & 47 \\
Significant Other & 26 & 10 & 0 & 72 \\
Fibromyalgia & 21 & 15 & 0 & 58 \\
Major Depression & 17 & 13 & 2 & 53 \\
Generalized Anxiety Disorder & 18 & 12 & 2 & 56 \\
Phobias (all types) & 7 & 23 & 2 & 19 \\
Panic Disorder & 3 & 27 & 2 & 11 \\
Post-Traumatic Stress Disorder & 2 & 32 & 2 & 6 \\
Any Psychiatric Disorder & 26 & 10 & 2 & 69 \\
Raynaud & 6 & 30 & 1 & 17 \\
Irritable Bowel Syndrome & 3 & 33 & 1 & 8 \\
\hline
\end{tabular}

Note. Descriptive characteristics of the syndrome and presence or absence of associated disorders. Significant other: married or stable couple. Ratio: percentage of patients with the characteristic over the total available data (total minus missing data).

The overall comparison between the patients and the subjects groups was significant (MANOVA Wilk's Lambda $=.658 ; \mathrm{F}=7.43 ; \mathrm{df}=7 ; \mathrm{p}=.001$ ). Only dimensions were included th th stage, for collinearity reasons. ANOVA comparisons were then performed between the two groups for each TCI dimension and subdimension.

No significant differences were found for the dimension Novelty Seeking (NS). Harm Avoidance (HA) was significantly more elevated $(p=.001)$ in patients than in controls, but the only subdimension significantly higher was that which scores Fatigability $(\mathrm{p}=.001)$. The comparison between the sum of the three subdimensions remaining after elimination of Fatigability was not significant. A significant difference was observed for Reward Dependence and its subdimension Dependence. There was no difference at the dimension Persistence.

The Character dimension Self-Directedness (SD) and its subdimension Congruent Second Nature were shown significantly higher in patients than in controls. No difference was observed for the dimension Cooperativeness although its subdimension Helpfulness was significantly more elevated in patients. No difference was observed for Self-Transcendence (ST).

In the comparisons established after stratification by presence $(n=26)$ or absence $(n=10)$ of psychiatric diagnosis, only the results which differ from the comparison between controls and the CFS group as a whole are noted below. Patients with any comorbid axis-I psychiatric disorder significantly differed from the control group on: (1) more Anticipatory Worry (a dimension of HA); (2) less Spiritual Acceptance (a dimension of ST). Patients without any comorbid psychiatric disorder differed from controls on: (1) more Exploratory Excitability (a dimension of NS); (2) more Reward Dependence; (3) more Self-Directedness and more on all its subdimensions (statistical differences or trends); (4) more Empathy and more Helpfulness (dimensions of Cooperativeness). 
Published in : Journal of Chronic Fatigue Syndrome (2007), vol. 14, pp. 55-68 DOI:10.1300/j092v14n01_06

Status : Postprint (Author's version)

TABLE 2. Comparison of dimensions and subdimensions of the TCI, stratification by presence or absence of psychiatric diagnosis

\begin{tabular}{|c|c|c|c|c|c|c|c|c|c|c|c|}
\hline & \multicolumn{2}{|c|}{$\begin{array}{c}\text { CTRL } \\
(n=72)\end{array}$} & \multicolumn{2}{|c|}{$\begin{array}{c}\text { CFS } \\
(n=36)\end{array}$} & \multicolumn{2}{|c|}{$\begin{array}{l}\text { CFS vs. PSY } \\
(n=26)\end{array}$} & \multicolumn{2}{|c|}{$\begin{array}{l}\text { CFS vs. NoPSY } \\
\qquad(n=10)\end{array}$} & \multirow{2}{*}{$\begin{array}{c}\text { CTRL vs. } \\
\text { CFS-All } \\
p\end{array}$} & \multirow{2}{*}{$\begin{array}{c}\text { CTRL vs. } \\
\text { CFS-PSY } \\
p\end{array}$} & \multirow{2}{*}{$\begin{array}{c}\begin{array}{c}\text { CTRL } \\
\text { vs. } \\
\text { CFS-NoPSY }\end{array} \\
p\end{array}$} \\
\hline & Mean & SD & Mean & SD & Mean & SD & Mean & SD & & & \\
\hline Age (years) & 38.1 & 7.7 & 37.2 & 8.6 & 37.2 & 7.7 & 37.6 & 11.0 & ns & ns & ns \\
\hline Novelty Seeking & 17.1 & 5.2 & 17.1 & 5.5 & 16.8 & 5.8 & 17.6 & 4.5 & ns & ns & ns \\
\hline Exploratory excitability & 4.9 & 2.3 & 5.4 & 2.4 & 5.0 & 2.5 & 6.5 & 2.5 & ns & ns & .047 \\
\hline Impulsiveness & 4.1 & 2.3 & 3.4 & 2.4 & 3.4 & 2.5 & 3.4 & 2.5 & ns & ns & ns \\
\hline Extravagance & 4.7 & 1.9 & 4.6 & 1.7 & 4.8 & 1.5 & 3.9 & 1.9 & ns & ns & ns \\
\hline Disorderliness & 3.1 & 1.7 & 3.7 & 1.4 & 3.6 & 1.4 & 3.8 & 1.6 & ns & ns & ns \\
\hline Harm Avoidance & 18.2 & 7.3 & 23.0 & 6.7 & 24.1 & 6.4 & 20.2 & 7.3 & .001 & .001 & ns \\
\hline Anticipatory worry & 4.9 & 2.7 & 5.6 & 2.8 & 6.2 & 2.6 & 3.9 & 2.8 & ns & .029 & ns \\
\hline Fear of uncertain & 4.7 & 1.9 & 4.9 & 1.7 & 5.1 & 1.8 & 4.5 & 1.3 & ns & ns & ns \\
\hline Shyness with strangers & 4.3 & 2.4 & 4.6 & 2.3 & 4.6 & 2.2 & 4.4 & 2.6 & ns & ns & ns \\
\hline Fatigability & 4.3 & 2.3 & 7.9 & 1.4 & 8.1 & 1.2 & 7.4 & 1.7 & .001 & .001 & .001 \\
\hline Reward Dependence & 16.1 & 3.4 & 17.8 & 3.4 & 17.2 & 3.1 & 19.0 & 3.7 & .030 & ns & .020 \\
\hline Sentimentality & 7.4 & 1.6 & 7.9 & 1.6 & 7.9 & 1.7 & 7.9 & 1.4 & ns & ns & ns \\
\hline Attachment & 5.2 & 2.1 & 5.1 & 2.6 & 4.8 & 2.5 & 5.8 & 2.8 & ns & ns & ns \\
\hline Dependence & 3.4 & 1.5 & 4.7 & 1.2 & 4.4 & 1.2 & 5.3 & .8 & .001 & .001 & .001 \\
\hline Persistence & 4.9 & 1.7 & 5.0 & 1.8 & 5.2 & 1.9 & 4.6 & 1.3 & ns & ns & ns \\
\hline Self-Directedness & 29.1 & 8.0 & 32.4 & 7.7 & 30.6 & 7.6 & 37.0 & 6.0 & .046 & ns & .001 \\
\hline Responsibility & 4.8 & 2.2 & 5.6 & 2.3 & 5.1 & 2.2 & 6.6 & 2.2 & ns & ns & .019 \\
\hline Purposefulness & 5.1 & 1.8 & 5.9 & 1.7 & 5.7 & 1.5 & 6.3 & 2.0 & ns & ns & $(.074)$ \\
\hline Resourcefulness & 3.3 & 1.4 & 3.8 & 1.6 & 3.5 & 1.7 & 4.4 & .8 & ns & ns & .027 \\
\hline Self-acceptance & 7.8 & 2.2 & 8.1 & 2.1 & 7.6 & 2.0 & 9.3 & 1.9 & ns & ns & $(.053)$ \\
\hline Congruent second nature & 7.9 & 2.7 & 9.1 & 2.7 & 8.6 & 2.6 & 10.4 & 2.4 & .029 & ns & .001 \\
\hline Cooperativeness & 32.7 & 6.0 & 34.7 & 4.3 & 34.3 & 4.2 & 36.0 & 4.6 & ns & ns & .097 \\
\hline Social acceptance & 6.7 & 1.7 & 7.0 & 1.3 & 7.1 & 1.1 & 6.9 & 1.9 & ns & ns & ns \\
\hline Empathy & 5.1 & 1.4 & 5.5 & 1.4 & 5.2 & 1.5 & 6.3 & .9 & ns & ns & .016 \\
\hline Helpfulness & 6.1 & 1.3 & 6.7 & 1.2 & 6.4 & 1.3 & 7.0 & 1.0 & .047 & ns & .034 \\
\hline Compassion & 7.7 & 2.7 & 8.4 & 1.9 & 6.5 & 1.8 & 8.3 & 1.9 & ns & ns & ns \\
\hline Principled & 7.0 & 1.6 & 7.2 & 1.4 & 7.0 & 1.5 & 7.5 & 1.1 & ns & ns & ns \\
\hline Self-Transcendence & 11.7 & 5.3 & 10.6 & 5.4 & 10.4 & 5.9 & 10.8 & 4.2 & ns & ns & ns \\
\hline Self-forgetfulness & 3.9 & 2.1 & 4.3 & 2.7 & 4.6 & 2.9 & 3.5 & 1.4 & ns & ns & ns \\
\hline Transpersonal identification & 3.4 & 1.7 & 2.9 & 2.1 & 3.0 & 2.2 & 2.3 & 1.8 & ns & ns & $(.079)$ \\
\hline Spiritual acceptance & 4.4 & 2.9 & 3.4 & 2.7 & 2.8 & 2.3 & 5.0 & 3.1 & ns & .014 & ns \\
\hline
\end{tabular}

Note. MANOVA; statistical trends are in parentheses. CTRL: control patients; CFS-Psy: CFS patients with at least one psychiatric axis-I disorder; CFS-noPsy: CFS patients without any psychiatric disorder. 


\section{DISCUSSION}

The comparisons performed here showed a relatively a specific personality profile in patients with CFS versus control subjects. If Fatigability is extracted from Harm Avoidance, the dimension is not significantly different. Patients with CFS were mostly shown to be (slightly) more Dependent than controls, and more perfectionnist. Patients without psychiatric comorbidity even showed higher scores than controls on usually well-considered subdimensions such as those included in dimensions Self-Directedness and Cooperativeness. As personality is supposed to exist before the beginning of the disorder symptoms and to remain stable along life, it is believed that it does not play a major role in the predisposition, the precipitation or the perpetuation of chronic fatigue. A higher level of Harm Avoidance had also been observed in another study on TPQ (Christodoulou et al., 1999). In that study, subdimensions Shyness with Strangers and Fatigability were significantly higher and the largest difference on the means were at subdimension Fatigability. Given that fatigue is the patients' main symptom, it is no surprise that this score was elevated, but it may not solely represent psychological aspects of personality. That same study also showed significant differences for subdimensions Anticipatory Worry and Shyness with Strangers. However, the scores of control subjects were somewhat low for Harm Avoidance in this study $(9.78 \pm 1.02)$, which may have increased the differences with the patient group. In the present study, it is remarkable that with the subtraction of the subdimension fatigability, the pool of remaining three subdimensions of Harm Avoidance was not significantly different. There was more Anticipatory Worry in the CFS patients with psychiatric diagnoses than in the control group.

It has also been shown that Harm Avoidance could be correlated with higher levels of anxiety and depression (Strakowski et al., 1995), although no difference was shown before and after treatment in a depressed patients sample (Marijnissen et al., 2002). The comparison with the study by Christodoulou et al.(1999) is also interesting since the anxious and depressed patients were excluded in that study, whereas patients in the present study were not, in accordance with the CDC criteria. Having more anxious and depressed patients could have increased the differences in Harm Avoidance, which was not the case. The similarity of these results are in favor of a limited influence of anxiety and depression on Harm Avoidance.

Reward Dependence and its subdimension Dependence was shown to be higher in patients (with or without psychiatric comorbidity). It could indicate a higher affective dependency to social values or a need for support (Pélissolo and Lépine, 1997). Dependence could thus be a characteristic of the personality of CFS patients.

Self-Directedness was shown to be higher in patients than in controls, especially those without psychiatric comorbidity. The subdimension Congruent Second Nature was also higher and indicates behaviors linked to values and long-term goals. It concerns the control of impulsive reactions and volatile ideas, favoring perfectionnism. A trend toward perfectionnism was also noted in the White et al. study (2000). Higher scores than in control subjects on this dimension are somewhat counterintuitive since it explores the ability to be responsible, self-acceptant, with higher control on behavior. 
The dimension Cooperativeness was not shown to differ between patients and controls as a whole. If anything, the results point towards more Empathy and more Helpfulness than in controls, which is also counter intuitive.

The main limitation of this study is the relatively small number of subjects and patients. Also, tertiary care patients samples do not fully represent unselected population.

This study, using for the first time Cloninger's TCI, shows a personality profile of patients with CFS, which only slightly differs from controls. If personality is not a major issue in CFS, then the etiology of the syndrome must be found in other areas, such as axis-I psychiatric disorders, or most likely, in more somatic domains. 


\section{References}

American Sleep Disorders Association. International Classification of Sleep Disorders (ICSD-Revised) 1997. Rochester, Minn.

Cho HJ, Skowera A, Cleare A, Wessely S (2006). Chronic fatigue syndrome: an update focusing on phenomenology and pathophysiology. Curr. Opin. Psychiatry; 19(1): 67-73.

Christodoulou C, Deluca J, Johnson SK, Lange G, Gaudino EA, Natelson BH (1999). Examination of Cloninger's basic dimensions of personality in fatiguing illness: Chronic fatigue syndrome and multiple sclerosis. J. Psychosom. Res.; 47(6): 597-607.

Cloninger CR (1986). A unified biosocial theory of personality and its role in the development of anxiety states. Psychiatr. Dev.; 3:167-226.

Cloninger CR, Przybeck TR, Svrakic DM, Wetzel RD (1994). The Temperament and Character Inventory (TCI): A guide to its development and use. St. Louis (MO): Center for psychobiology of personality, Washington University.

Cloninger CR, Svrakic DM, Przybeck TR (1993). A psychobiological model of temperament and character. Arch. Gen. Psychiatry; 50:975-90.

Cloninger R (1987). A systematic method for clinical description and classification of personality variants. A proposal. Arch. Gen. Psychiatry; 44(6):573-88.

Eysenck HJ (1967). The Biological Basis of Personality, Carles C. Thomas, Ed. Springfield, CO.

Eysenck HJ (1990). Biological basis of personality. In L.A. Pervin (Ed). Handbook of Personality: Theory and Research. New York, Guildford Press: 244-76.

Fischler B (1999). Review of clinical and psychobiological dimensions of the chronic fatigue syndrome: Differentiation from depression and contribution of sleep dysfunctions. Sleep Med. Rev.; 3(2):131-46.

Frances A (1982). Categorical and dimensional systems of personality diagnosis: A comparison. Compr. Psychiatry; 23(6):516-27.

Frost R, Marten P, Lahart C, Rosenblate R (1990). The dimensions of perfectionnism. Cognit. Ther. Res.; 14:449-68.

Fukuda K, Straus SE, Hickie I, Sharpe MC, Dobbings JG, Komaroff A and the International Chronic Fatigue Syndrome Study Group (1994). The chronic fatigue syndrome: A comprehensive approach to its definition and study. Ann. Intern. Med.; 121:953-9.

Furberg H, Olarte M, Afari N, Goldberg J, Buchwald D, Sullivan PF (2005). The prevalence of self-reported chronic fatigue in a U.S. twin registry. J. Psychosom. Res.; 59(5):283-90.

Gallagher AM, Thomas JM, Hamilton WT, White PD (2004). Incidence of fatigue symptoms and diagnoses presenting in UK primary care from 1990 to 2001. J. R. Soc. Med.; 97(12):571-5.

Hansenne M, Le Bon O, Gauthier A, Ansseau M (2001). Belgian Normative Data of the Temperament and Character Inventory. Eur. J. Psychol. Assess.; 17:56-62.

Johnson SK, DeLuca J, Natelson BH (1996). Personality dimensions in the chronic fatigue syndrome: A comparison with multiple sclerosis and depression. J. Psychiat. Res.; 30:9-20. 
Johnson SK, DeLuca J, Natelson BH (1999). Chronic fatigue syndrome: Reviewing the research findings. Ann. Behav. Med.; 21(3):258-71.

Le Bon O, Fischler B, Hoffmann G, Murphy JR, De Meirleir K, Cluydts R, Pelc I (2000). How significant are primary sleep disorders and sleepiness in the chronic fatigue syndrome. Sleep Res. Online; 3(2): 43-8.

Le Bon O, Staner L, Tecco J, Pull C, Pelc I (1998). Questionnaire de personnalité tridimensionnel (TPQ): Validation chez une population contrôle francophone. L'Encéphale; 24:40-5.

Maquet D, Demoulin C, Crielaard JM (2006). Chronic fatigue syndrome: A systematic review. Ann. Readapt. Med. Phys.; 49(6):418-27.

Marijnissen G, Tuinier S, Sijben AE, Verhoeven WM (2002). The temperament and character inventory in major depression. J. Affect. Disord.; 70(2):219-23.

Millon C, Salvato F, Blaney N, Morgan R, Mantero-Atienza E, Klimas N, Fletcher M (1989). A psychological assessment of chronic fatigue syndrome/chronic EpsteinBarr virus patients. Psychol. Health; 3:131-41.

Pélissolo A, Lépine J-P (1997). Traduction française et premiéres études de validation du questionnaire de personnalité TCI. Ann. Méd. Psychol.; 155(8):497-508.

Pepper CM, Krupp LB, Friedberg F, Doscher C, Coyle P (1993). A comparison of neuropsychiatric characteristics in chronic fatigue syndrome, multiple sclerosis, and major depression. J. Neuropsychiatry Clin. Neurosci.; 5:200-5.

Rosenberg M (1965). Society and the Adolescent Self-Image. Princeton, NJ: Princeton Univ. Press.

Russo J, Katon W, Sullivan M, Clark M, Buchwald D (1994). Severity of somatization and its relationship to psychiatric disorders and personality. Psychosomatics; 35:546-56.

Schmaling KB, Jones JF (1996). MMPI profiles of patinets with chronic fatigue syndrome. J. Psychosom. Res.; 40:67-74.

Strakowski SM, Dunayevich E, Keck PE, McElroy SL (1995). Affective state dependence of the Tridimensional Personality Questionnaire. Psychiatry Res. 28; 57(3): 209-14.

Van Hoof E, De Meirleir K (2005a). Chronic Fatigue syndrome and myalgic encephalomyelitis: Are both conditions on the same continuum? North Am. J. Psychol.; 7(2):189-204.

Van Hoof E, De Meirleir K (2005b). The Influence of Chronic Fatigue Syndrome on the Personality Profile: A Case Report. J. Chronic Fatigue Syndr.; 12(3):61-73.

Weir PT, Harlan GA, Nkoy FL, Jones SS, Hegmann KT, Gren LH, Lyon JL (2006). The incidence of fibromyalgia and its associated comorbidities: A population-based retrospective cohort study based on International Classification of Diseases, 9th Revision codes. J. Clin. Rheumatol.; 12(3):124-8.

Wessely S, Chadler T, Hirsch S, Wallace P, Wright D (1996). Psychological symptoms, somatic symptoms and psychiatric disorder in chronic fatigue and chronic fatigue syndrome: A prospective study in primary care. Am. J. Psychiatry; 153:1050-9.

White C, Schweitzer R, et al. (2000). The role of personality in de development and perpetuation of chronic fatigue syndrome. J. Psychosom. Res.; 48(6):515-24. 